\title{
GPU Cost Estimation for Load Balancing in Parallel Ray Tracing
}

\author{
Biagio Cosenza ${ }^{1}$, Carsten Dachsbacher ${ }^{2}$ and Ugo Erra ${ }^{3}$ \\ ${ }^{1}$ Universität Innsbruck, Austria \\ ${ }^{2}$ Karlsruhe Institute of Technology, Germany \\ ${ }^{3}$ Università della Basilicata, Italy \\ cosenza@dps.uibk.ac.at,dachsbacher@kit.edu,ugo.erra@unibas.it
}

Keywords: $\quad$ Ray Tracing, Image-based Techniques, Parallel Rendering, GPU.

\begin{abstract}
Interactive ray tracing has seen enormous progress in recent years. However, advanced rendering techniques requiring many million rays per second are still not feasible at interactive speed, and are only possible by means of highly parallel ray tracing. When using compute clusters, good load balancing is crucial in order to fully exploit the available computational power, and to not suffer from the overhead involved by synchronization barriers. In this paper, we present a novel GPU method to compute a cost map: a per-pixel cost estimate of the ray tracing rendering process. We show that the cost map is a powerful tool to improve load balancing in parallel ray tracing, and it can be used for adaptive task partitioning and enhanced dynamic load balancing. Its effectiveness has been proven in a parallel ray tracer implementation tailored for a cluster of workstations.
\end{abstract}

\section{INTRODUCTION}

Ray tracing algorithms (Gla89) model physical light transport by shooting rays into the scene with the ultimate goal of producing photorealistic images. Considerable efforts have been made in order to investigate new ways to reduce the high computational demands of ray tracing. Recent advances in ray tracing include exploiting coherence between neighboring pixels with packet traversal (Wal04), ray sorting (GL10), frustum traversal (RSH05), and fast updating of the acceleration data structure for animated scenes (ZHWG08; Cos08). Thanks to recent improvements in both software and hardware, Whittedstyle ray tracing reaches interactive frame rates on CPUs (ORM08) and GPUs (LGS ${ }^{+}$09). However, if we want to provide more realism in the produced images, e.g. by computing global illumination, we need to drastically increase the number of secondary rays. Even though several optimization strategies allow a certain amount of interactivity, the use of complex rendering techniques and shading algorithms, shadows, reflections and other global illumination effects drastically increase computation. The number of rays grows from less than one million of typically coherent rays, up to several millions (sometime even billions) of mostly incoherent rays. In this context, distributing ray tracing among several workers, i.e. multiple CPUs or GPUs, is a viable solution to reach interactive frame rates.
In this paper, we describe a GPU-based approach for an efficient estimate of the per-pixel rendering cost. The approach uses information available in the G-Buffer generated by widely used deferred shading techniques. By using the per-pixel rendering cost, we are able to estimate the rendering time of any part of the image generated by Whitted-style ray tracing or path tracing.

A scenario where this approach would be useful is a parallel ray tracing on clusters, where one master node (equipped with a GPU) is responsible for distributing tiles of an image (i.e. tasks) to several worker nodes (equipped with multi-core CPUs or GPUs) which perform ray tracing computation. In order to have a good load balancing, the master node tries to equally distribute tasks to each node minimizing the response time. Then, using our approach to estimate the ray tracing cost of a tile of an image, we can further improve task partitioning and dynamic load balancing strategies.

We validate our idea by implementing a parallel ray tracing system based on Whitted-style ray tracing and path tracing that exploits the estimate of the per-pixel rendering cost for balancing and distributing rendering tasks across workers in a network. We perform the following steps to balance the rendering load:

1. Compute a per-pixel, image-based estimate of the rendering cost, called cost map. 

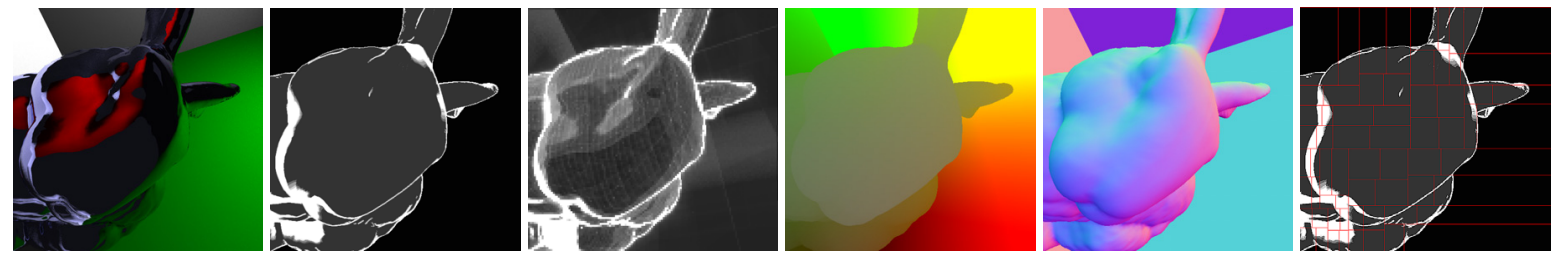

Figure 1: Left to right: the ray-traced image, the GPU-based estimation of the rendering cost, the actual packet-based rendering cost, position and normals in eye-space (used for the cost estimation), and using our method for an adaptive tiling of the image for parallel rendering.

2. Use the cost map for subdivision and/or scheduling in order to balance the load between workers.

3. A dynamic load balancing scheme improves balancing after the initial tiles assignment.

We show that the per-pixel cost estimate enables good load balancing while maintaining a small number of tiles, thus allowing high rendering performance even with slow networks.

\section{PREVIOUS WORK}

Ray tracing parallelization approaches can be classified into two main categories: image parallel decomposition and scene geometry parallel decomposition (SGPD), sometime referred as data parallel (CR02). With image parallel methods, each worker is responsible for a region of the image (e.g. a pixel or a tile), while the scene data is usually (but not necessarily) replicated in the memory of each node. On the contrary, in $S G P D$ methods, each worker is responsible for a part of the scene, while rays propagate among the nodes. An image parallel approach is typically the better solution for scenes that can be stored in a single node as in our case.

Early works in parallel ray tracing that reached interactive performance used massively parallel shared memory supercomputers (Muu95). Current hardware trends in processor designs are turning towards multi-core architectures and wide vector instructions. Manta (BSP06) is an interactive ray tracing system combining a high level of parallelism with modern packet-based acceleration structures. It uses a multithreaded scalable parallel pipeline in order to exploit parallelism on multi-core processors. Similarly, (GS08) focuses on exploiting the massive parallelism of multi-core hardware.

Ray tracing on distributed memory systems. Developing interactive ray tracing for distributed memory systems is an intricate process. Extending a renderer's architecture to a cluster of workstations requires implementing several components, such as a high-performance communication layer and an efficient dynamic load balancer. Without these techniques, the overhead of the communication causes poor scalability and performance penalties. Commodity-based clusters offer a cost-effective solution to speed up ray tracing, and many parallel rendering frameworks as GigaWalk (BSGM02) or VR MantaJuggler (OSR09) already support networked workstations.

Wald et al. (WSBW01) used coherent ray tracing techniques in distributed memory architectures. Their system used a central server that takes care of load balancing and stores the whole scene, but they were able to render large and complex models at interactive rates by using a two level BSP for per-node caching of geometry. Later, several works improved these techniques in order to render massively complex models (WDS04; DSW07). Distributed Shared Memory systems (DSM) offer a virtual distributed memory address space in which each node of a cluster has access to shared memory in addition to each node's nonshared private memory. DeMarle et al. (DGP04), and more recently Ize et al. (IBH11), presented a state-ofthe-art read-only DSM ray tracer tailored for cluster hardware. Our work is different in two aspects: We focus on high-quality rendering using more involved algorithms, such as path tracing, for scenes that can be stored in a single node. Moreover our parallelization approach is based on the message passing paradigm. Budge et al. (BBS $\left.{ }^{+} 09\right)$ introduced a system that enables the rendering of globally illuminated images of large, complex scenes by using a hybrid CPU/GPU algorithm on a cluster. They developed an efficient out-of-core data-management layer and coupling this with an application layer containing a path tracer.

Load balancing. The major challenge of parallel ray tracing on clusters is load balancing. In particular, if a rendering system is subject to a barrier synchronization point (e.g., in synchronous rendering), then the slowest task will determine the overall performance. Achieving a good load balance is not trivial and can be achieved using two main strategies: trying to equally partition tasks, or using dynamic work assignment. Also choosing the number of tiles to subdivide the image into (i.e. task granularity) is non- 
trivial. For example, while a higher number of tiles facilitates load balancing in a dynamic work assignment strategy, it also results in a high number of communication, which is critical in networks with high latency.

Researchers have proposed many strategies for addressing load balancing in this context. Heirich and Arvo (HA98) discussed the importance of dynamic load balancing for ray tracing in interactive settings. Further related work examines the importance of the subdivision granularity ( $\mathrm{Pla02}$ ), and suggests adaptive subdivision to balance the workload $\left(\mathrm{CCD}^{+} 08\right)$.

Although a demand driven centralized balancing scheme achieves a well balanced workload, it involves significant master-to-worker communication which becomes a bottleneck when network transmission delay and the number of workers increase. A decentralized load balancing scheme, such as work stealing (BL99) or work redistribution, eliminates the communication bottleneck thus improving performance and scalability.

DeMarle et al. (DPH ${ }^{+}$03; DGBP05) implemented a decentralized load balancing scheme based on work stealing. It is important to notice that in their implementation, task migration is done at the beginning of the next frame (frame-to-frame steals), and the synchronization bottleneck at the master node is hidden by an asynchronous task assignment. Ize et al. (IBH11) use a master dynamic load balancer with a work queue comprised of large tiles which are given to each node (the first assignment is done statically and is always the same), and each node has its own work queue where it distributes sub-tiles to each render thread.

More generally, the problem of load balancing is very important for parallel rendering and visualization and many solutions have been introduced, as for instance using a kd-tree to divide the image into tiles of equal cost (MWMS07) or a cost estimation based on the intersected primitives (Mue95).

Because of the higher load imbalance with more involved rendering techniques (e.g. path tracing), and because of the GPU availability on the master node, we balance workload in a more effective way: We implemented four different balancing strategies, based on adaptive tiling, dynamic load balancing and cost prediction, and we combine them with a multithreading parallelization based on tile buffering.

Rendering Cost Evaluation. Several factors affect the rendering cost in ray tracing, such as scene size, resolution, rendering technique, coherence between rays, material properties, and the choice of the acceleration data structure. In our context shading algorithms affect the number of secondary rays traced into the scene, and are the major cause load imbalance among different pixels in the image (Figure 3).

Some techniques known in literature tackle the problem of estimating the rendering cost. Gillibrand et al. (GLDC06) suggest that an approximate render cost can be generated from a rasterized scene preview. Profiling techniques are approaches where the computation time of a small number of samples (or pixels) is measured in order to have a coarse approximation of the rendering cost. While using a distributed memory architecture, profiling is typically done on the master rather than on the workers. If performed on the workers, sample measures have to be sent back. Another issue is that tracing few incoherent rays is slow. For these reasons, we did not use profiling in our work. Beyond image-space approaches, rendering cost estimation has been explored even for SGPD (RKC98).

An important remark is that our workload differs from (BWS03) in two aspects: First, we use Whitted ray tracing and path tracing instead of Instant Global Illumination; second, our test scene exhibits high variance in shaders and geometry. Both contribute to a high workload imbalance per pixel (as shown by the real cost map in Figure 12), which is challenging for system scalability. Our workload is more similar to $\left(\mathrm{CDS}^{+} 06\right)$, where a parallel selective renderer is used for physically-based rendering.

\section{THE COST MAP}

In this section, we show how to obtain the cost map, i.e. the image-based, per-pixel cost estimate of the rendering process (see Figure 1 and Figure 2). First, we define the problem statement and related approaches. Then, we introduce a GPU approach capable of quickly computing an approximate cost map, and finally analyze the cost estimate error.
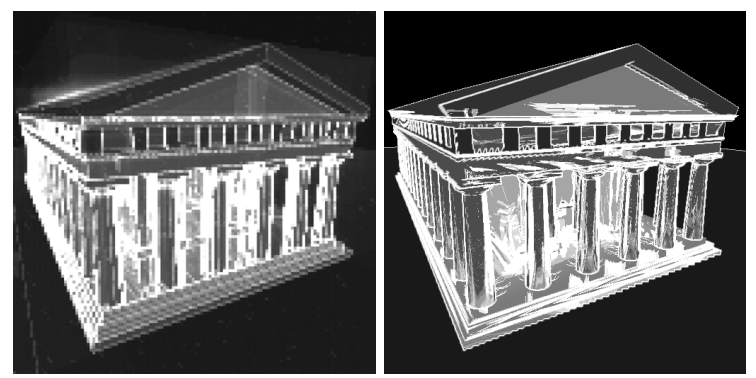

Figure 2: A comparison of the real cost (left) and our GPUbased cost estimate (right). 


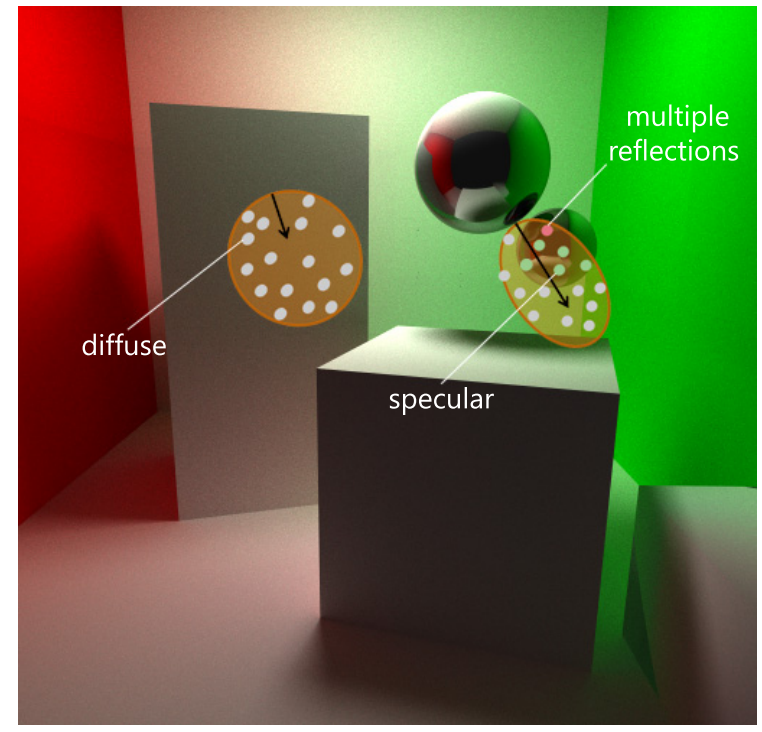

Figure 3: Estimating the rendering cost: diffuse surfaces typically have lower cost (in particular for Whitted-style ray tracing), while specular surfaces generate more secondary rays thus causing higher rendering cost. Regions where multiple reflections occur are typically more expensive and can be found by a search in image-space. When using Monte Carlo-based techniques, the image-space search can be adapted according to the specular coefficient of the surface (see the sphere in the images).

\subsection{The GPU-based Cost Map}

To compute the cost map quickly on the GPU, we make use of G-buffers known from deferred shading (Har04) and image-space sampling. The underlying idea is that it is often possible to detect potentially expensive areas, e.g. with multiple interreflections by solely performing a search for such geometric configuration using image-space information.

Algorithm 1 describes our image-based technique, assuming that the properties of each pixel belonging to a visible surface are available.

We assign a certain basic cost to each pixel $P_{i}$ depending on its material, e.g. the cost for evaluating the BRDF model. Next, if the surface is reflective, we perform an image space search in order to detect potentially expensive areas, e.g. where multiple interreflections are likely. To this end, we create a set of samples which are used to obtain the information about the surfaces from the G-buffer. We use an initially uniformly distributed set of sampling points which is transformed before sampling (line 5). This transformation is computed according to the reflection properties of the surfaces (mainly glossiness), the rendering technique (Whitted-style raytracing, path tracing etc.) and oriented along the reflection vector $R$ projected into image space. Intuitively, the pattern

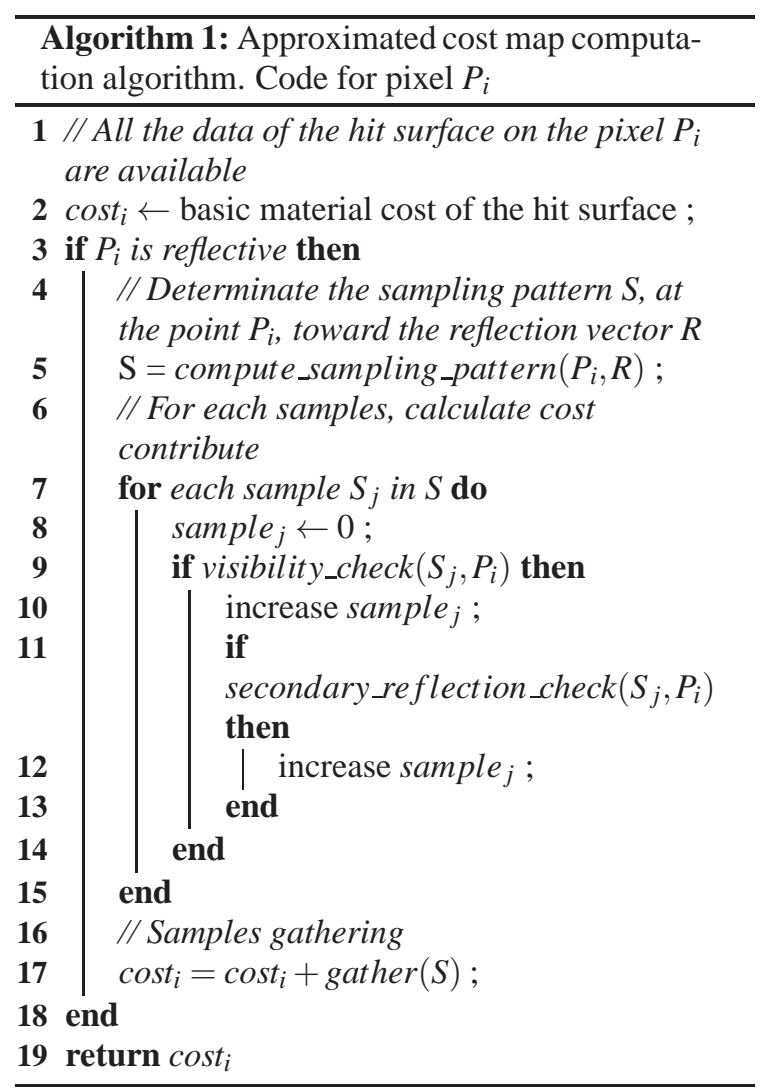

is scaled to become more narrow for surfaces with higher specularity, and positioned at the surface point $P_{i}$ in question and oriented along the projection of its reflection vector (Figure 3).

For every sample $S_{j}$, we retrieve its surface location and orientation from the G-Buffer. Next, we perform a test to detect the sample cost: We test if $P_{i}$ and $S_{j}$ are mutually front-facing (line 9). Later, a test detects if the sampled surface is reflective (line 11). If the surface at $S_{j}$ is reflective, and the lobe of specular reflection of $S_{j}$ points towards $P_{i}$ then we detected a region with a potentially high number of interreflections, and further increase the cost estimate. After the sampling phase, we gather the contributions from all samples.

Sampling pattern. The sampling pattern evaluated by compute ampling $_{p}$ attern $\left(P_{i}, R\right)$ (see Figure 4 ) is randomly generated (a) and scaled (b) according to the surface properties. Essentially, the pattern is translated (c) and rotated (d) to align with the reflection vector $R$. Step (b) depends on the material as well as the rendering technique. In particular, we distinguish a wider sampling pattern for Lambertian and glossy surfaces for path tracing, but the pattern collapses to a line for Whitted-style ray tracing and 


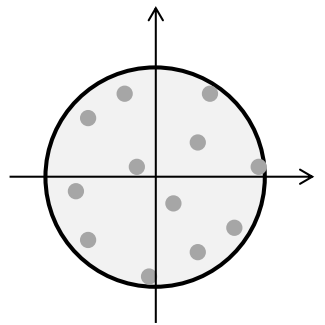

(a)

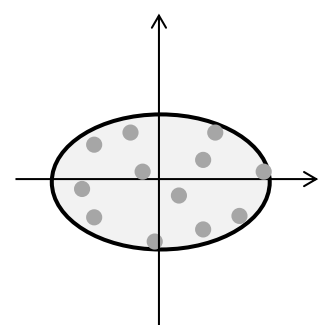

(b)

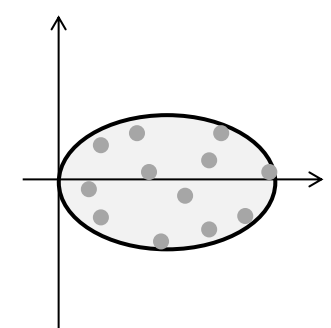

(c)

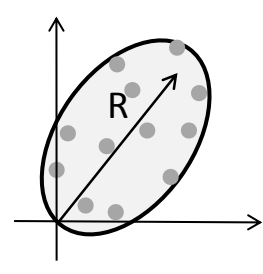

(d)

Figure 4: Sampling pattern. The sampling patterns used in the cost map generation algorithm. At first, an uniformly distributed set of points is generated (a). According to the shading properties, the pattern is scaled (b) and translated to the origin (c). In the last step, it is transformed according to the projection of the reflection vector in the image plane(d).

for path tracing using a perfect mirror material. The sampling for the cost map computation is performed only once per pixel, not recursively.

Sample gathering. Once sampling has been performed, we gather the contributions from all samples. This step is somewhat correlated to the rendering technique. In particular, the final cost may be computed in two ways: by summing up the sample contributions, or by taking their maximum. The first approach is used in path tracing, where we suppose that secondary rays spread along a wide area. In the contrary, when using Whitted ray tracing, all samples belong to one secondary ray and we conservatively estimate the cost by taking the maximum.

Edge detection. Real-time ray tracers use bundles of coherent rays, called ray-packets, to achieve realtime performance on CPUs (Wal04; ORM08). Whenever packet-based ray tracing is used, packet splitting raises the cost because of the loss of coherence between rays. This occurs at depth discontinuities that we detect by using a simple edge-detection filter on the G-Buffer. By further increasing the cost estimate at edges, we account for the impact of packet splitting. We experienced that this extra cost is significant only with Whitted ray tracing.

Implementation details. The algorithm has been implemented in a two pass shader. In the first pass, we render the scene to a G-Buffer using multiple render targets to store data. For every pixel, we store the position, the normal, and a value indicating if the surface is reflective, for the first visible surface seen from the camera (Figure 3). We also store three additional values: The basic shader cost, the specular coefficient and the Phong exponent. In the second pass, we generate the cost map using the image-space information stored in the G-Buffer. The basic value has been used in several points of the algorithm (i.e. lines 2 and 10). The other values both are required in the sampling phase (line 5 and Figure 4). Details about rendering parameters are shown in Table 1 . The memory re- quired by the algorithm amounts to three screen-sized textures used by the G-Buffer (in our implementation, three $512^{2}$ floating-point RGBA textures, i.e. $12 \mathrm{MB}$ ).

The cost map generation is fast: Whereas both CPU and GPU computation take less than $1 \mathrm{~ms}$, the most expensive task is the data transfer between GPU and CPU (5-6 ms). However, this time is spent by the master node in barrier, i.e. when preparing the cost map before distributing the work load. For that reason, this time is further hidden using an pipelined prediction optimization (details in Section 5).

\subsection{Cost Estimation Error Analysis}

The resulting cost map is obviously approximate. We analyzed the error in order to understand where and why the estimate is accurate and where not. Because of the use of ray-packets, our analysis is performed at packet-level instead of pixel-level. First, we measure the difference between the approximate cost and the real cost (packet-based error) for each ray-packet. Second, we analyze difference maps of real and approximate cost to be thoroughly aware about where the estimate is less accurate. In the subsequent sections, we analyze the performance of a parallel ray tracing system, evaluating how much the cost map enables better task distribution to several worker nodes by using two balancing techniques.

Five test scenes (Figure 5) have been used to evaluate the estimate of the cost map. In Figure 6, we show the error distribution of the (packet-based) difference between the real computing time and the estimated one. The analysis shows that the estimate is quite accurate, and errors usually lead to an underestimation, caused by the off-screen geometry problem. Because our strategy works on a rasterized scene preview, our algorithm only works on visible geometry. Nevertheless, reflected rays may fall in geometry not present in the frame buffer. When this happens, the algorithm is not able to detect a reflected surface 


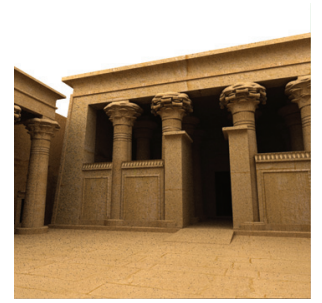

Kalabsha Temple

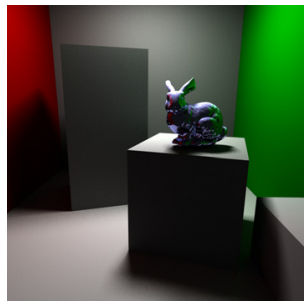

Cornell box

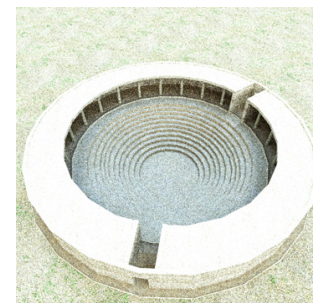

Ekklesiasterion

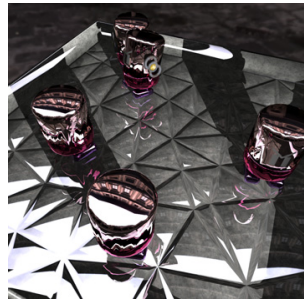

Toasters

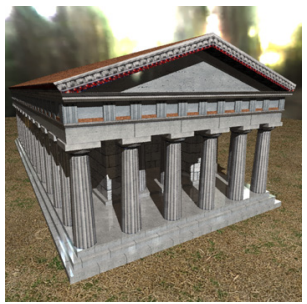

Paestum Temple

Figure 5: Our test scenes. Rendering parameters are shown in Table 1.

and the resulting calculated cost is under-estimated in respect to the real one (Figure 7).

For instance, in the case of the Cornell box scene, the cost estimation is quite accurate: the $86 \%$ of the predictions fall in the first approximation interval $(+/-5 \%$ of the real packet time). Note that the $87 \%$ of the predictions are under-estimated (error $\geq 0$ ). In the contrary, the Ekklesiasterion scene is less accurate: only the $57 \%$ have a good approximation. Here, $84 \%$ of the predictions are under-estimated. Figure 12 shows real cost and GPU-calculated cost maps, and the difference between real and approximate cost.

Note that we can reduce the off-screen problem by computing the cost map for an extended image plane to capture surfaces outside the current view. The use of the A-buffer would further guarantee that the whole geometry is available on the render buffer (Car84), and it requires only a fixed amount of GPU memory that increases linearly with image space size (YHGT10). One limitation of our implementation is that the scene should fit into the GPU memory. However, because we use the GPU only for the cost map computation (i.e. does not affect the final rendering), one could easily use low-resolution meshes or level-of-detail approaches for the GPU cost map estimation, which might then be slightly less accurate.

\section{LOAD BALANCING}

In this section, we describe how we use the cost map for two different load balancing strategies. In order to exploit the cost map, we use a Summed Area Table (Cro84) created on the GPU. Summed Area Tables (SATs) allow us to compute the sum of values in a rectangular region of an image in constant time. A SAT of the cost map allows us to directly compute the cost estimate for an image tile. Given a cost map of dimension $n \times n$ (where $n$ is the number of pixel per dimension), we can compute a SAT directly on the GPU in $O(\log n)$ time $\left(\mathrm{HSC}^{+} 05\right)$.

SAT Sorting. An immediate use of the SAT is to compute the cost of a tile after subdividing the image in equally sized tiles. Next, we can sort the tiles for decreasing cost, assuring that computationally more expensive tiles are scheduled before cheaper ones. The reason for this approach is that dynamic load balancing typically works better if nodes work on more expensive tasks at first, and task transfers or steals are performed for smaller tasks afterward (e.g. chunk scheduling (GKKA03)).

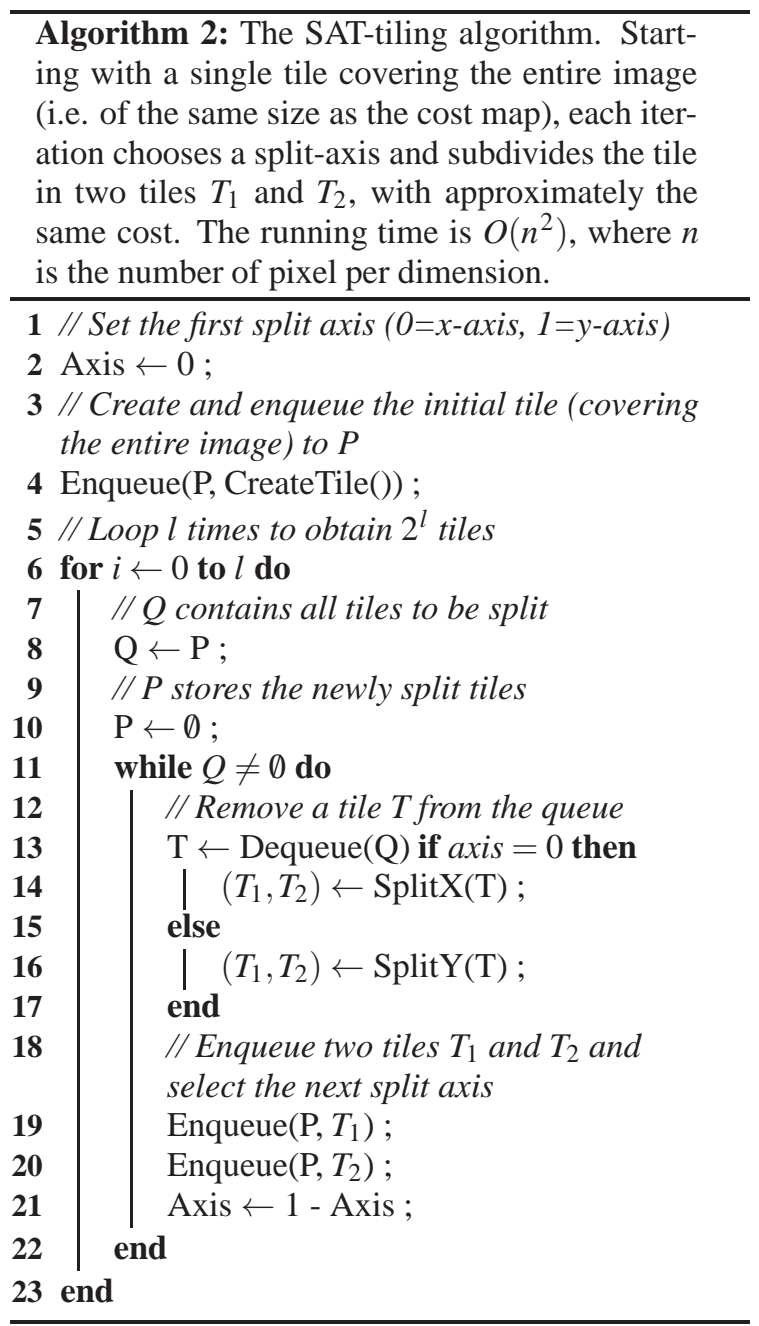



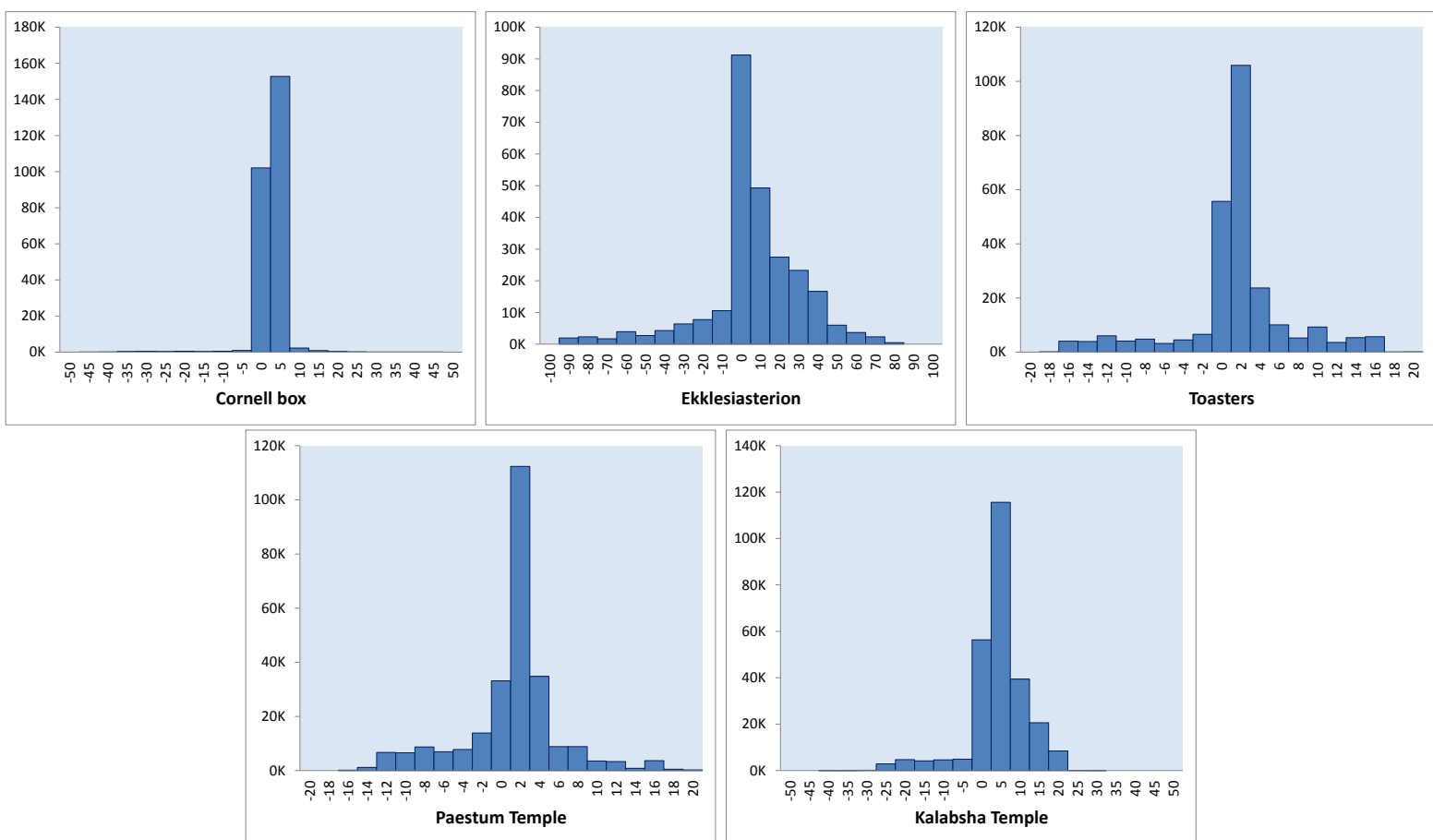

Figure 6: Error distribution of the estimation. Each packet-based rendering time is subtract from the cost map estimate, for the same corresponding packet of pixels. The x-axis shows the difference in error intervals, from negative values (left, over-estimation) to positive ones (right, under-estimation). The y-axis plots error occurrences for each error interval. Tests have been performed on one Intel Pentium IV CPU $3.40 \mathrm{GHz}$ with $2048 \mathrm{~KB}$ cache size, averaged for a walk through of few frames in the scene.

SAT Adaptive Tiling. We can alternatively use the SAT to determine an adaptive subdivision of the image space into tiles of roughly equal cost. Our adaptive subdivision algorithm can be seen as a weighted kd-tree split using the SAT to locate the optimal splits.

In our implementation we use two temporary deques (double-ended queues), $P$ and $Q$, and the cost map $C$ and the number of iterations $l$ as input. During subdivision, we use $Q$ to store the current tiles to be split, and $P$ to store the tiles that have already been split. The resulting subdivision of our algorithm is well balanced and all tiles exhibit almost equal cost (see Algorithm 2).

\section{PARALLEL RAY TRACING}

In this section, we briefly discuss the main challenges of our parallel ray tracing system: the tile-topacket mapping, the dynamic load balancing, and further optimizations. In order to validate the use of the cost map, we implemented Whitted-style ray tracing and path tracing.

Our implementation of the work assignment, scheduling, and dynamic load balancing is decoupled from the ray tracing implementation that runs on the individual worker. We base our ray tracing implementation on Manta (BSP06), which we extended with new image traversal algorithms, load balancers, shaders, and by adding new components. In particular, our parallel code is hidden behind the traversal logic, with a master-side and a worker-side component. The first is responsible for the prediction and assignment, and implements a GPU-based rendering system with programmable shaders. The latter hides the CPU-based ray tracing system and the dynamic distributed load balancer. A material table is in charge of linking the ray tracer shading information with the GPU-cost map generation. As we use static scenes, we employ precomputed kd-trees built with a $\mathrm{SAH}$ metric as acceleration data structure.

Tile-to-Packet Mapping. As we discussed before, we use a ray tracer which bundles coherent rays together to ray-packets. The packeting size is usually set to a value that enables exploit SIMD units and optimize data locality. Recent approaches encourage the usage of large ray packets for Whitted-style ray tracing (ORM08).

The use of two different levels of parallelism, one being the packets of the ray tracer, and one being the 

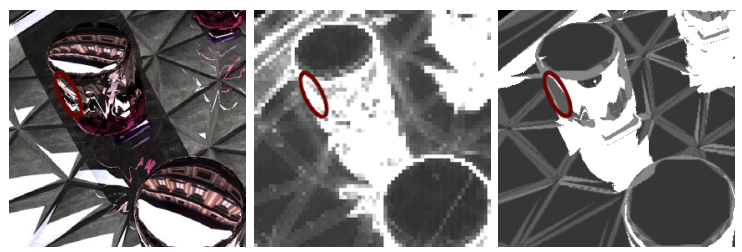

Figure 7: Off-screen geometry problem. The image indicates an area where secondary rays fall outside geometry in the rendering buffer (a), hence raising the cost of these pixels (b). Our GPU technique under-estimates the cost of this area (c).

splitting of the image into tiles, raises the problem of how to map a tile to packets. The optimal packet size mainly depends on the scene, the acceleration data structure, and the hardware architecture. Similarly, a parallel distributed memory system has an optimal task (i.e. tile) size that depends on the ratio of computation to the amount of communication, being critical in systems like a cluster of workstations. Fixing the same task size for both with an one-to-one approach does not reach optimal performance of the whole system. A one-to-one approach also complicates, and limits, the exploitation of the cost map for adaptive subdivision. Our system uses a one-to-many approach instead: each tile is subdivided into packets of fixed optimal size, e.g. each tile is subdivided in packets of $8 \times 8$ rays per packet. Ize et al. (IBH11) used a similar two-level load balancer in their DSM-based implementation, but in our work, the first level load balancer is based on work stealing (i.e. distributed load balancing).

Work Stealing. Our parallel system performs inframe steals to improve the load balancing computed from the cost estimation. Note that a perfect cost map would make work stealing superfluous; however, this cannot be expected from an image-space estimation. Our work stealing implementation follows the scheme suggested in (BL99) where each worker has a queue ${ }^{1}$ : each worker first processes his own tasks starting from the top of his queue. When the queue is empty, workers start stealing tasks from another randomly chosen worker (Figure 8). Although dynamic load balancing is distributed, first task assignment is centralized. All tiles are assigned at the beginning of the frame by the master node, using prefetching to hide latency (i.e. by assigning all the tiles at once, without any on demand request from the workers) and assuring fairness (i.e. preventing starvation of MPI processes). Then the dynamic distributed load bal-

\footnotetext{
${ }^{1}$ We adopt the terminology of (BL99) using the word queue. However, as our work stealing algorithm performs operations in both the top and the bottom of the queue, the correct term would be deque.
}

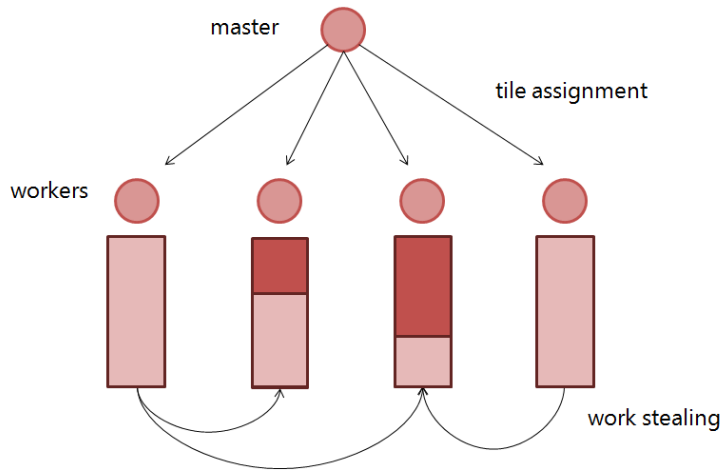

Figure 8: A master node is responsible for the first task assignment. Using work stealing idle workers search among the other workers in order to find unprocessed tiles.

ancing algorithm takes care of an initially unbalanced work distribution. Further optimizations in the work stealing protocol can save communication when, for instance, two nodes send crossed steal requests (i.e. avoiding to send two negative ack messages). An important aspect when implementing in-frame steals is to take care of the frame synchronization barrier. A node entering into stealing mode performs steal requests until a new frame starts. The new frame message, however, is sent by the master node without a guarantee for order-preserving and delivery, i.e. it may happen that a node at frame $f+1$ receives an old steal request sent by a node at the frame $f$. We solve this problem by adding the frame number to the steal request and steal ack messages.

Further optimizations. On each node, the ray

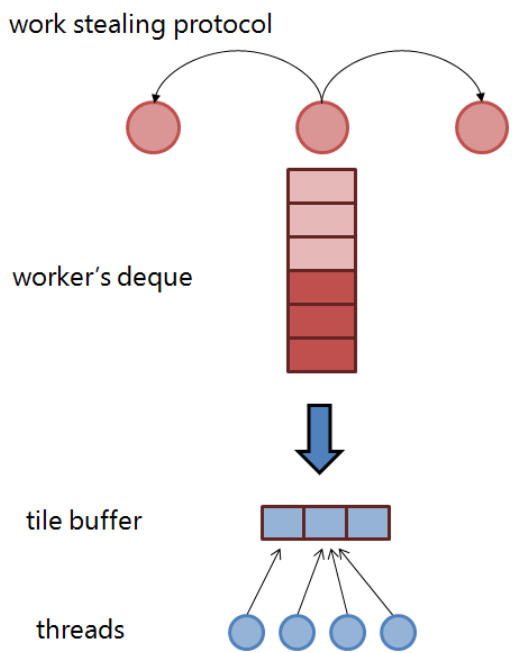

Figure 9: Multi-threading with tile buffering. A small number of tiles moves from the queue to the buffer. This allows separating the work stealing algorithm, working on the queue, from the multi-threading parallelization, working on the tile buffer. 
tracing itself can also be parallelized via multiple threads and ray packets, e.g. if multi-core CPUs and SIMD instruction sets are available. On top of Manta, our distributed parallel architecture introduces the queue of tiles as an additional element to support work stealing. Moreover, a tile buffer has been introduced to efficiently support multi-threading. Figure 9 shows how the tile buffer is integrated with the work stealing queue.

We utilize MPI as a means to exchange data between nodes. Our system hides latency by implementing task prefetching and using asynchronous data transfer where possible. At the beginning of each frame, the camera position and a list of prefetched tiles is sent to each worker.

The overhead introduced for generating the cost map, the SAT and to compute the tiling causes a longer barrier. We hide this overhead by means of a pipelined prediction: after the task assignment for the frame $f$, when all workers are busy, the master node starts computing the prediction for the frame $f+1$. The overall architecture is still synchronous, whereas just the prediction phase is computed asynchronously.

\section{RESULTS}

We ran several benchmarks on a test platform consisting of a cluster of workstations equipped with Intel six-core Xeon X5650 CPUs running at $2.7 \mathrm{GHz}$ and 24 GB DDR3 ECC RAM. Nodes are interconnected with an Infiniband network. The master and visualization node is equipped with an NVidia GeForce GTX 570. The cost map computation has been implemented using OpenGL and GLSL. Running the master and the worker node on a single host may reduce performance. For this reason, we used a specific visualization host as the master node.

Test scenes. All images have been rendered at a resolution of $1024 \times 1024$ pixels, with 128 tiles and a maximum recursion depth of 4 , resulting in highly varying rendering cost in regions with interreflections. Pipelined prediction has been enabled in all tests. In order to study the impact of our techniques, we used five different test scenes (Figure 5), having different rendering parameters, rendering techniques and overall workload: The first three scenes use path tracing and thus are computationally more expensive. The first is the Kalabsha temple. For each pixel, we apply a jitter pattern of 128 rays/pixel, shooting 134.2 million of primary rays. We used the same parameters for the second scene, a Cornell box with a reflective Bunny and an area light. The Ekklesiasterion scene contains an ancient Greek building. For this scene, we used 32 rays/pixel. The other two test scenes were rendered using Whitted ray tracing with respectively 8 and 1 rays/pixel. The Toaster and PoseidoniaPaestum temple both present a large number of reflective surfaces. Table 1 shows ray-packet size, the number of primary rays, and the rendering parameters used for each test scene. We performed scalability tests by using up to 16 workers; each worker uses 6 threads.

For each test scene, we show results using 4 different balancing approaches: A simple unbalanced approach without dynamic load balancing and using equally sized tiles (Regular without WS); a regular approach using work stealing for load balancing (Regular); an adaptive approach using the SAT of the cost map and work stealing (SAT Adaptive); a sortingbased approach using SAT and work stealing. For all approaches, there is an initial tiles assignment phase where each node gets a set of tiles that are close in image space. Table 2 shows performance for 16 workers and shows the parallel speedup and efficiency.

An analysis of the number of steal transfers is shown for all the test scenes in Figure 10. This analysis is helpful to understand how different tiling algorithms work and, once an initial tile set is assigned, how balancing algorithms integrate with work stealing algorithms.

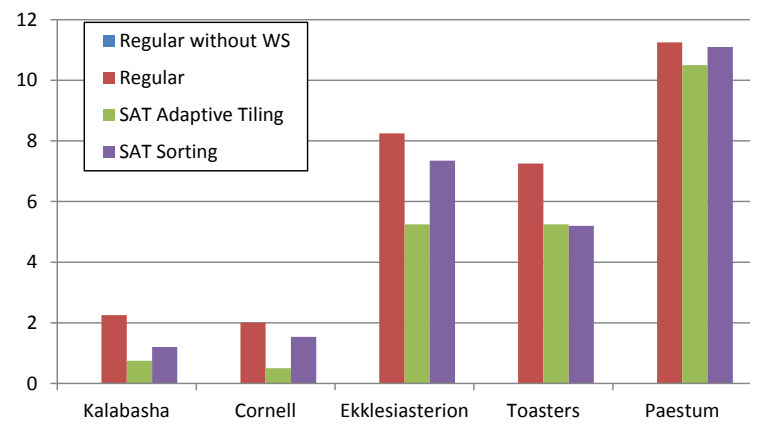

Figure 10: Steal transfers. The graph shows the average number of steal transfers performed during our test, using 4 workers. Note that a node may steal even more than one tile per frame. Note, the regular approach does not use work stealing.

\section{DISCUSSION}

Our analysis of the results focuses on the effectiveness of the load balancing techniques utilized (SAT Adaptive Tiling and SAT Sorting), their correlation with the approximation of the cost map, and scalability.

SAT Adaptive Tiling. Our results show that the Adaptive Tiling is similar in performance to the Regu- 


\begin{tabular}{|l|ccccc|}
\hline Scene & Kalabsha temple & Cornell box & Ekklesiasterion & Toasters & Paestum temple \\
\hline \hline \# triangles & 4529462 & 69495 & 3346 & 11141 & 13556 \\
Rendering tech. & path tracing & path tracing & path tracing & whitted & whitted \\
Rays/pixel & 128 jitter & 128 jitter & 32 jitter & 8 jitter & 1 \\
Packet size & 8 & 8 & 16 & 16 & 32 \\
Primary rays & $134.2 \mathrm{M}$ & $134.2 \mathrm{M}$ & $33.6 \mathrm{M}$ & $8.4 \mathrm{M}$ & $1.0 \mathrm{M}$ \\
\hline Sampling pattern & wide pattern & wide pattern & wide pattern & collapsed & collapsed \\
& & & & to a line & to a line \\
Edge detection & no & no & no & yes & yes \\
Samples gathering & sum & sum & sum & max & max \\
\hline
\end{tabular}

Table 1: Summary of the parameters used for rendering and cost map computation. The packet size is the optimal value for each test scene.

\begin{tabular}{|l|ccccc|}
\hline Scene & Kalabsha temple & Cornell box & Ekklesiasterion & Toasters & Paestum temple \\
\hline \hline Regular without WS & 5.285 & 2.901 & 0.765 & 0.067 & 0.0126 \\
Regular & 4.862 & 2.783 & 0.750 & 0.066 & 0.0112 \\
SAT Adaptive Tiling & 4.889 & 2.680 & 0.745 & 0.064 & 0.0108 \\
SAT Sorting & 4.542 & 2.548 & 0.638 & 0.059 & 0.0102 \\
\hline 1 worker & 70.639 & 40.005 & 9.391 & 0.932 & 0.154 \\
\hline Speedup* & $13.4-15.6 \times$ & $13.8-15.7 \times$ & $12.3-14.7 \times$ & $13.9-15.8 \times$ & $12.2-15.1 \times$ \\
Efficiency* & $83-97 \%$ & $86-98 \%$ & $76-91 \%$ & $86-98 \%$ & $76-94 \%$ \\
& $+16 \%$ & $+13 \%$ & $+19 \%$ & $+13 \%$ & $+23 \%$ \\
\hline
\end{tabular}

Table 2: Performance comparison for our five test scenes; timings are given in seconds per frame. We report speedup and also efficiency which describes the fraction of the time that is being used by the processors. Tests have been performed with 16 workers and multi-threading. (*) Speedup and efficiency is shown for Regular without WS and SAT Sorting approach

lar approach. However, it never outperforms the $S A T$ Sorting approach. The steal transfers analysis (Figure 10) reveals additional information. An important issue is that the number of steal transfers of adaptive tiling is often the lowest. This means that (1) the initial tile assignment provided by the adaptive tiling is more balanced than the regular one; (2) work stealing does not work well with such kind of (almost balanced) workload. In fact dynamic load balancing, in our case the work stealing strategy, rivals adaptive tiling since both try to balance the workload: a fine grained balancing with dynamic techniques requires more expensive tiles at the beginning and the cheaper ones at the end; but in contrast to that, adaptive tiling aims to equally balance cost between tiles.

The adaptive tiling performance is the worst for scenes with higher approximation error where (e.g. the Ekklesiasterion). In fact, this scene shows the least accuracy (see Figure 6). This indicates that the accuracy required by an adaptive approach is critical, and in general a very accurate cost map is required in order to significantly increase rendering speed.

SAT Sorting. The use of the SAT for sorting tiles always improves performance. Contrary to adaptive tiling, this technique does not require an exact estimation of the rendering cost: it just needs a correct tile ordering. In particular, the use of the SAT Sort- ing strategy, combined with a distributed load balancing algorithm, helps assuring a good load balance. The combined use of both techniques, sorting and dynamic load balancing, also achieves a good scalability with the number of workers (Table 2). The rationale behind is that adaptive approaches benefit from the information how much costly a tile is than another one, while sort-based approaches only requires to understand which one is bigger.

Scalability. In order to determine how our system scales with the number of distributed workers, we ran a scalability test with $1,4,8$, and 16 workers. Figure 11 shows the scalability for the Cornell box test scene. Results indicate that the SAT Sorting approach provides higher scalability and is particularly useful with high workloads. The improvement of the SAT Sorting strategy, compared to a naïve balancing-unaware approach is about $13-23 \%$ with 16 workers. Efficiency is always superior to $95 \%$ with Whitted style ray tracing test scenes. Instead, using path tracing we have slightly lower performance, with an efficiency of at least $91 \%$ with 16 workers.

Even though work stealing is a popular approach to distributed dynamic load balancing, its performance is not well understood yet. The effectiveness of our sorting-based approach raises new interesting applications in the context of massively parallel pro- 
cessing. In our system, work stealing is particularly efficient when we have more than 8 workers. Hence, it seems to be a perfect candidate for today's and future massively parallel systems. Our steal analysis also shows that the number of steal transfers is lower with high workloads. We suppose that this is related to the fixed number of tiles size and we plan to investigate how to beneficially change this number according to the workload. Increasing the quality, e.g. using more samples per pixel and using path tracing, only slightly affects the scalability of the system.

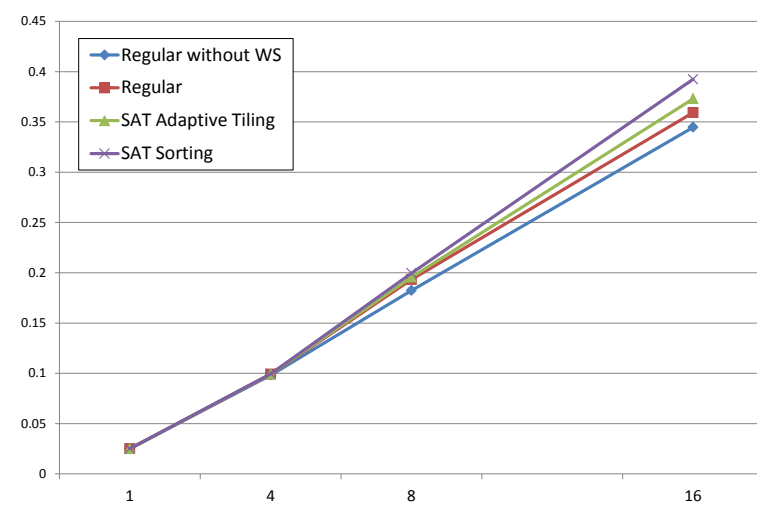

Figure 11: Scalability for up to 16 workers measured for the Cornell box and path tracing. Timings are in frames per second.

\section{CONCLUSION}

In this paper, we described a GPU-based algorithm used to compute a per-pixel ray tracing cost estimate. The proposed approach, based on deferred shading and image sampling, is fast and requires only a fixed amount of GPU memory that increases linearly with image size and produces a good approximation of the real computation time. Moreover, our approach could use any type of cost estimate; for instance it could also be applied to a cost map produced by using the ray tracing algorithm itself (i.e. profiling).

In order to validate our idea, we implemented a parallel ray tracing system for distributed memory architectures based on Whitted-style ray tracing and path tracing. Together with our ray tracing system, we also presented two methods that exploit the cost map in order to speed up performance: SAT Adaptive Tiling uses the cost map in order to subdivide the image in tile with the same cost and SAT Sorting instead exploits the cost map using a dynamic load balancing, and sorts tiles according to their cost. Our results indicate that while the SAT Adaptive tiling is more sensitive to the cost map approximation, SAT Sorting is always the best approach. It fits well into dynamic load balancing and provides good scalability for multiple workers.

In future work, we plan to introduce an automatic cost map tool to facilitate the tuning of the cost map generation algorithm. We would like to see how effective these techniques can be for GPU-based ray tracing implementations (e.g. Nvidia OptiX $\left(\mathrm{PBD}^{+} 10\right)$ employs a three-tiered dynamic load balancing approach on multi-GPUs). Moreover, we are interested in evaluating the effectiveness of the techniques while having models that do not fit the GPU memory, hence by using a simplification technique for the cost map computation. With increasingly more computational power for commodity hardware and the availability of multicore architectures, we believe that similar balancing techniques will become of growing interest in the next future.

\section{ACKNOWLEDGEMENTS}

Part of this work was funded by the Austrian Science Foundation FWF (DK+CIM, W1227) and also by the Austrian Ministry of Science BMWF as part of the UniInfrastrukturprogramm of the Research Platform Scientific Computing at the University of Innsbruck.The first author initiated this work at the Visualization Research Center, Universität Stuttgart, and has been partially funded by a DAAD Scholarship and a HPC-EUROPA2 project(228398). Carsten Dachsbacher acknowledges support from the Intel Visual Computing Institute, Saarbruecken.

\section{REFERENCES}

B. Budge, T. Bernardin, J. Stuart, S. Sengupta, K. Joy, and J. Owens. Out-of-core Data Management for Path Tracing on Hybrid Resources. In Eurographics, 2009.

R. D. Blumofe and C. E. Leiserson. Scheduling multithreaded computations by work stealing. Journal of ACM, 46(5):720-748, September 1999.

W. V. Baxter, III, A. Sud, Naga K. Govindaraju, and D. Manocha. GigaWalk: Interactive Walkthrough of Complex Environments. In Eurographics workshop on Rendering, EGRW, pages 203-214, 2002.

J. Bigler, A. Stephens, and S.G. Parker. Design for Parallel Interactive Ray Tracing Systems. In IEEE Symposium on Interactive Ray Tracing, pages 187 -196, sept. 2006.

C. Benthin, I. Wald, and P. Slusallek. A Scalable Approach to Interactive Global Illumination. Computer Graphics Forum, 22(3):621-630, 2003.

L. Carpenter. The A-buffer, an antialiased hidden surface method. In ACM SIGGRAPH, pages 103-108, 1984.

B. Cosenza, G. Cordasco, R. De Chiara, U. Erra, and V. Scarano. Load Balancing in Mesh-like Computations using Prediction Binary Trees. In Symposium on Parallel and Distributed Computing (ISPDC), pages 139-146, 2008. 
A. Chalmers, K. Debattista, V. Sundstedt, P. Longhurst, and R. Gillibrand. Rendering on Demand. In EGPGV, pages 9-17, 2006.

B. Cosenza. A Survey on Exploiting Grids for Ray Tracing. In Eurographics Italian Chapter Conference, pages 89-96, 2008.

A. Chalmers and E. Reinhard. Pratical Parallel Rendering. AKPeters, 2002.

F. C. Crow. Summed-area Tables for Texture Mapping. In 11th annual Conference on Computer Graphics and Interactive Techniques, SIGGRAPH, pages 207-212, 1984.

D. E. DeMarle, C. P. Gribble, S. Boulos, and S. G. Parker. Memory Sharing for Interactive Ray Tracing on Clusters. Parallel Comput., 31(2):221-242, February 2005.

D. E. DeMarle, C. P. Gribble, and S. G. Parker. MemorySavvy Distributed Interactive Ray Tracing. In EGPGV, pages 93-100, 2004.

D. E. DeMarle, S. Parker, M. Hartner, C. Gribble, and C. Hansen. Distributed Interactive Ray Tracing for Large Volume Visualization. In IEEE Symposium on Parallel and Large-Data Visualization and Graphics, PVG, pages 12-, 2003.

A. Dietrich, A. Stephens, and I. Wald. Exploring a Boeing 777: Ray Tracing Large-Scale CAD Data. IEEE Comput. Graph. Appl., 27(6):36-46, November 2007.

A. Grama, G. Karypis, V. Kumar, and Gupta A. Introduction to Parallel Computing, 2nd edition. Pearson Addison Wesley, 2003.

K. Garanzha and C. T. Loop. Fast Ray Sorting and BreadthFirst Packet Traversal for GPU Ray Tracing. Computer Graphics Forum, pages 289-298, 2010.

A. S. Glassner. An Introduction to Ray Tracing. Morgan Kaufmann, 1989.

R. Gillibrand, P. Longhurst, K. Debattista, and A. Chalmers. Cost prediction for global illumination using a fast rasterised scene preview. In AFRIGRAPH, pages 4148, 2006.

I. Georgiev and P. Slusallek. RTfact: Generic concepts for flexible and high performance ray tracing. In Interactive Ray Tracing, 2008. RT 2008. IEEE Symposium on, pages $115-122$, aug. 2008.

A. Heirich and J. Arvo. A Competitive Analysis of Load Balancing Strategies for Parallel Ray Tracing. Journal of Supercomputing, 12(1-2):57-68, January 1998.

S. Hargreaves. Deferred shading, 2004. Game Developers Conference Talks.

J. Hensley, T. Scheuermann, G. Coombe, M. Singh, and A. Lastra. Fast Summed-Area Table Generation and its Applications. Computer Graphics Forum, 24(3):547-555, 2005.

T. Ize, C. Brownlee, and C. D. Hansen. Real-Time Ray Tracer for Visualizing Massive Models on a Cluster. In EGPGV, pages 61-69, 2011.

C. Lauterbach, M. Garland, S. Sengupta, D. Luebke, and D. Manocha. Fast bvh construction on gpus. Computer Graphics Forum, pages 375-384, 2009.
C. Mueller. The sort-first rendering architecture for highperformance graphics. In Symposium on Interactive 3D graphics, I3D, pages 75-ff., 1995.

M. J. Muuss. Towards real-time ray-tracing of combinatorial solid geometric models. In BRL-CAD Symposium, 1995.

B. Moloney, D. Weiskopf, T. Mller, and M. Strengert. Scalable Sort-First Parallel Direct Volume Rendering with Dynamic Load Balancing. In Symposium on Parallel Graphics and Visualization (EGPGV), pages 45-52, 2007.

R. Overbeck, R. Ramamoorthi, and W.R. Mark. Large ray packets for real-time whitted ray tracing. In Interactive Ray Tracing, 2008. RT 2008. IEEE Symposium on, pages $41-48$, aug. 2008.

C. N. Odom, N. J. Shetty, and D. Reiners. Ray Traced Virtual Reality. In $I S V C$, pages 1031-1042, 2009.

S. G. Parker, J. Bigler, A. Dietrich, H. Friedrich, J. Hoberock, D. Luebke, D. McAllister, M. McGuire, K. Morley, A. Robison, and M. Stich. Optix: a general purpose ray tracing engine. ACM Trans. Graph., 29(4):66:1-66:13, July 2010.

T. Plachetka. Perfect load balancing for demand-driven parallel ray tracing. In International Euro-Par Conference on Parallel Processing, pages 410-419, 2002.

E. Reinhard, A. J. F. Kok, and A. Chalmers. Cost distribution prediction for parallel ray tracing. In Eurographics Workshop on Parallel Graphics and Visualisation, pages 77-90, September 1998.

A. Reshetov, A. Soupikov, and J. Hurley. Multi-level ray tracing algorithm. In ACM SIGGRAPH, pages 11761185, 2005.

I. Wald. Realtime Ray Tracing and Interactive Global Illumination. PhD thesis, Computer Graphics Group, Saarland University, 2004.

I. Wald, A. Dietrich, and P. Slusallek. An Interactive Outof-Core Rendering Framework for Visualizing Massively Complex Models. In Eurographics Symposium on Rendering, 2004.

I. Wald, P. Slusallek, C. Benthin, and M. Wagner. Interactive Distributed Ray Tracing of Highly Complex Models. In Eurographics Workshop on Rendering Techniques, pages 277-288, 2001.

J. C. Yang, J. Hensley, H. Grün, and N. Thibieroz. RealTime Concurrent Linked List Construction on the GPU. Comput. Graph. Forum, 29(4):1297-1304, 2010.

K. Zhou, Q. Hou, R. Wang, and B. Guo. Real-time KDtree construction on graphics hardware. In ACM SIGGRAPH Asia, pages 126:1-126:11, 2008. 

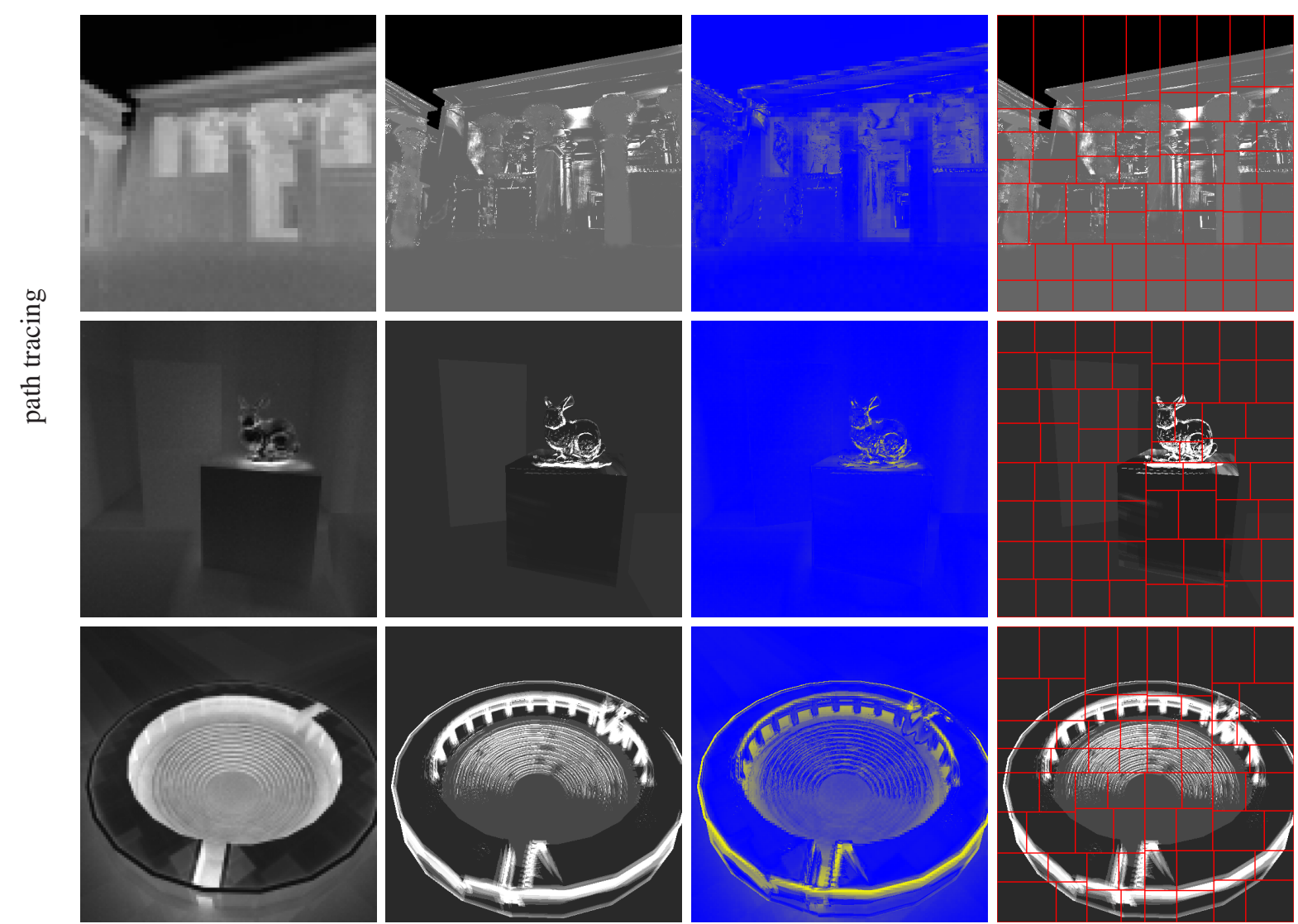

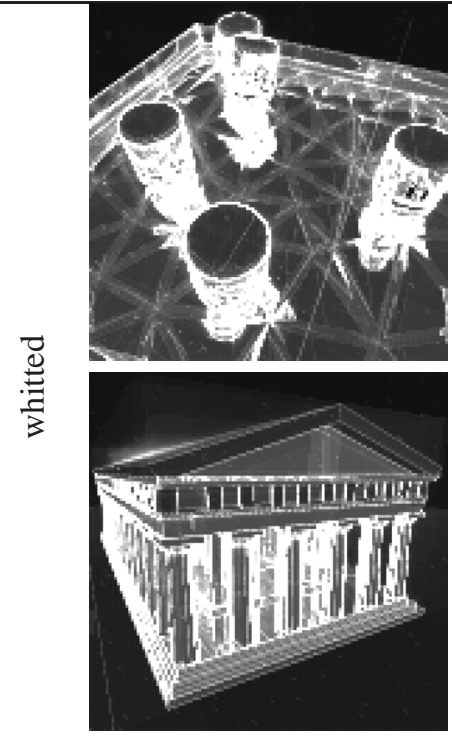

(a) real cost map
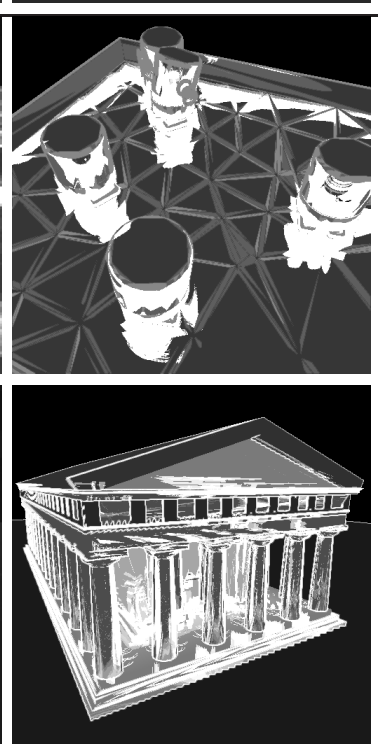

(b) GPU cost map
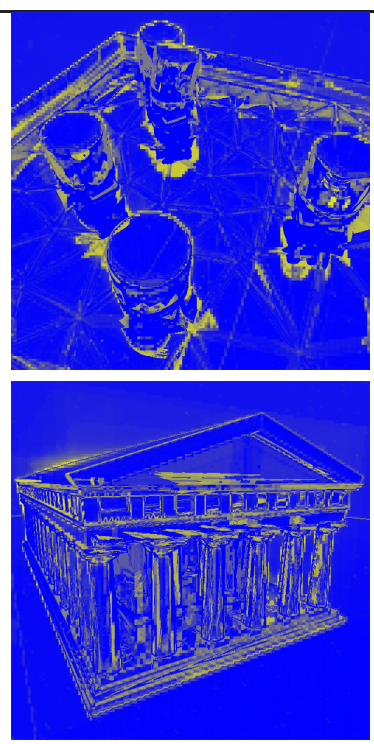

(c) difference
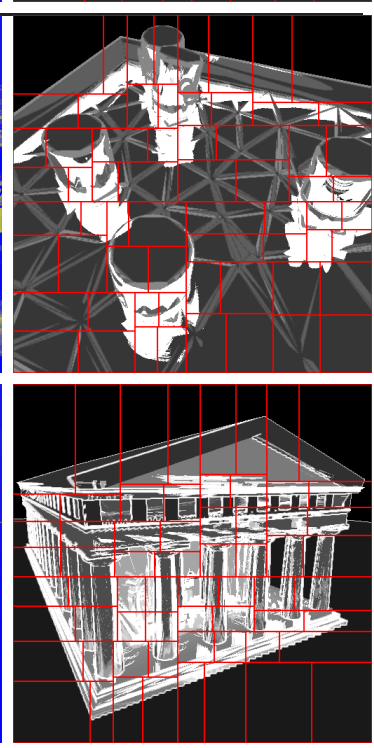

(d) adaptive tiling

Figure 12: Effectiveness of the cost map generation in different test scenes. For each scene, we show the real packet-based cost map based on timings (a); the GPU-based cost map estimate (b); an explanatory difference map mapped on a gradient: In blue areas estimation is precise, whereas yellow areas show less accuracy. (c); the resulting adaptive tiling (d). The cost maps are obtained mapping the cost of the pixel/packet into the range $[0,1]$. 